\title{
A Cardiac Electro-physiological Model Based Approach for Filtering High Frequency ECG Noise
}

\author{
MA Mneimneh, GF Corliss, RJ Povinelli \\ Marquette University, WI, USA
}

\begin{abstract}
With an increasing focus on automatic diagnoses of cardiac disease through ECG signals, de-noising techniques that do not introduce artifacts have become necessary. This paper proposes a model based approach for removing high frequency noise from ECG signals. The proposed modeling technique is based on the propagation of the electric waves over the cardiac tissue. The proposed approach models the crucial nodes as a difference between two sigmoid functions. The ECG signal is modeled as the sum of the activity at the SA node, AV node, Bundle branches, Purkenji fibers, and right and left ventricles. The model is adapted to the targeted ECG signal using a nonlinear least squares optimization technique. The proposed filtering approach is applied to randomly selected ECGs from the Long-Term ST database. A quantitative analysis is performed on simulated ECG signals perturbed with white noise with ST signal to noise ratios ranging from -25 to $5 \mathrm{~dB}$.
\end{abstract}

\section{Introduction}

The cardiac muscle is both electrically and mechanically active with the electrical system acting as a stimulator for the mechanical. Measurement through electrocardiogram (ECG) signals of the electrical activity of the heart provides important information for diagnosing cardiac disease. However, ECG signals are typically disturbed by noise from electric interference, electromyography, and baseline wandering. The filtering method presented in this paper focuses on removing electric interference and electromyography contamination from ECG signals.

Our approach models the generation of the ECG signal and uses this model based approach to filter high frequency noise from the ECG signal. The modeling approach is based on cardiac electrophysiology, where the ECG signal is generated from the modeling of the SA node, AV node, Bundle branches, Purkinje fibers, and left and right ventricles walls. The electrical activity of each of these components of the heart is estimated by the difference of two sigmoid functions. The model has the ability to characterize the P wave, PR segment changes, QRS complex, ST segment changes, and $\mathrm{T}$ wave. Therefore, diagnostic information contained in these components of the ECG signal is not lost by the filtering process.

\section{Background}

The simplest way to remove high frequency noise from an ECG signal is with a low pass filter. However, such filters introduce artifacts and/or temporal effects in the signal by removing the $\mathrm{Q}$ or $\mathrm{S}$ waves or by adding variations to the $\mathrm{T}$ wave end [1]. Additionally, current adaptive filtering methods that deal with high frequency signal denoising require a reference signal or a template model as an input [2].

Recently, Kestler applied a discrete wavelet transform and Wiener filter in order to suppress the high frequency noise in ECG signals [3]. Su et al. used a TranslationInvariant Wavelet De-Noising Method with Improved thresholding to de-noise ECG signals [4]. The drawback of these methods is that the right parameters for the wavelet decomposition are required to avoid adding temporal effects to the ECG signals or removing the Q-wave or S wave.

He et al. proposed the use of independent component analysis (ICA) for filtering ECG signals. He's approach used 12 ECG leads as inputs to ICA to extract the noise from the 12 lead signals [5]. The drawback of this approach is that all 12 signals are required, which might not be available in ambulatory recordings.

Clifford et al. used a Gaussian based approach to characterize the ECG signal [2]. However, this approach does not model ST and PR changes; thus some of the information is lost when filter ECGs that indicate ischemia or infarction.

\section{Data sets}

The proposed approach is applied to real and simulated ECG signals. The real signals are taken randomly from the Long-Term ST database . The LTST database consists of 86 long term (21 to 24 hour) ECG recordings sampled at $250 \mathrm{~Hz}$. The simulated signals are generated by the dynamical model developed by McSharrey and Clifford [6]. Several signal-to-noise ratios (SNR) of white, brown, and pink noise are added to the clean simulated signals to 
establish a quantitative evaluation of the proposed filtering approach.

\section{Methods}

The motivation for this modeling approach starts from the observation of the electric potential of a cardiac cell and specific groups of cells during the cardiac cycle. While substantial research has been done on the internal dynamics of the cardiac cell, we focus our model, for complexity and computational reasons, on characterizing the SA node, the AV node, bundle branches, Purkinje fibers, and left and right ventricles.

We have found the cardiac electrical cycle of these groups of cardiac cells is well modeled by the difference of two sigmoids. Figure 1 illustrates the output of an example model of a group of cardiac cells.

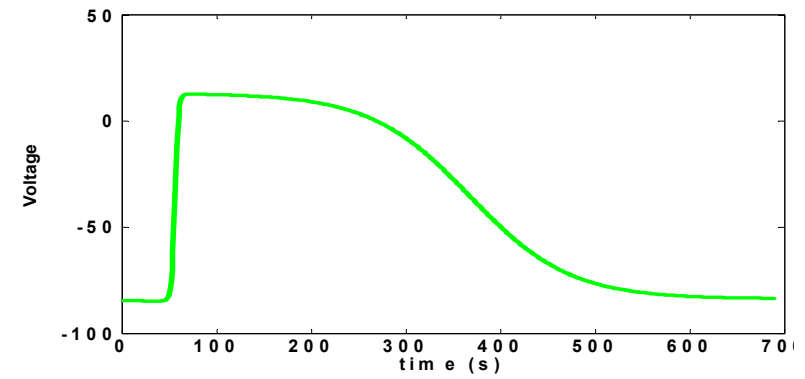

Figure 1: Proposed heart cell activity

While other mathematical structures such as polynomials may be used, there is a distinct advantage to the proposed difference of two sigmoids. Only five parameters are needed to model each cell group and these parameters correspond well to physiological characteristics of the heart and also to fiduciary points on the ECG. These parameters are the magnitude, inflection points, and slopes of the difference of two sigmoids. They correspond, respectively, to the magnitude of electrical activity, the inflection points of depolarization and repolarization, and the rate of potential change within the cell group.

By summing the potential difference of each cell group's electrical activity at the positive and negative terminals of each lead, the ECG signals are generated.

The following sections will present how the ECG wave features are generated. The features are the $\mathrm{P}$ wave, the PR segment, the $\mathrm{Q}$ wave, the $\mathrm{R}$ wave, and the $\mathrm{S}$ wave (QRS complex), ST segment, and T wave.

\subsection{Mathematical description}

As discussed above our cardiac model is composed of models of six key electrical components of the heart. Each component is modeled as the difference of two sigmoids.

$$
f\left(t, a_{1}, c_{1}, a_{2}, c_{2}, k\right)=k\left(\frac{1}{1-e^{a_{1}\left(t-c_{1}\right)}}-\frac{1}{1-e^{a_{2}\left(t-c_{2}\right)}}\right),
$$

where $k$ represent the translation of the wave in the direction of the y-axis, $a_{1}$ and $a_{2}$ control the rising slope, and $c_{l}$ and $c_{2}$ control the translation in the direction of the $\mathrm{x}$-axis.

The ECG signal is the sum of the potential activity of each cell group as measured at a lead. The signal measured at a lead is modeled as the difference between the signal arriving at the positive and negative electrodes of a lead. The resulting equation that estimates the ECG signal is:

$$
\hat{f}_{E C G}=\sum_{i \in[S A, A V, \text { Rven,Rvep,Lven, }, \text { Lve }]}\left(f_{i}^{+}-f_{i}^{-}\right),
$$

where $f_{i}^{+}$and $f_{i}^{-}$represent the cell group activity at the positive and negative electrodes for the SA node, AV node, bundle branches, Purkinje fibers, and left and right ventricles. $\hat{f}_{E C G}$ is the generated ECG signal.

In order to apply the proposed ECG model, the parameters of (2) are determined for a noisy ECG signal, such that sum squared error between the noisy ECG signal and the signal generated by the model are minimized:

$$
\text { Error }=\sum_{\text {signal }}\left(E C G-\hat{f}_{E C G}\right)^{2} \text {. }
$$

The error is minimized using a partially separable nonlinear least squares method. Since the proposed model is based on cardiac electrophysiology, the ECG signal is separated into atrial and ventricular activity. The atrial activity is divided into the P wave and PR segment modeled by the SA and AV nodes respectively. Therefore, the residual function of these features is:

$$
\text { error }_{\text {Atrial }}(x)=\sum_{i \in[S A, A V]}\left(\text { Atrial }_{\text {activity }}-\left(f_{i}^{+}-f_{i}^{-}\right)\right)^{2} \text {. }
$$

Similarly, the ventricular activity is divided into the Q wave, the R wave, the $\mathrm{S}$ wave, the ST segment and the $\mathrm{T}$ wave; which are represented in this case by the bundle branches, Purkinje fibers and left and right ventricles:

$$
\text { error }_{\text {vent }}(x)=\sum_{i \in[S A, A V, B b, P f, L V, R V]}\left(\text { Vent }_{\text {activity }}-\left(f_{i}^{+}-f_{i}^{-}\right)\right)^{2}
$$

The estimated clean ECG signal is the sum of the atrial and ventricular activity. The usage of the partially separable property enables us to lower the number of parameters to be estimated from 54 to 18 and 36 respectively; thus reducing computational time. The partially separable method takes into consideration the difference between the iso-electric line at the atrial and ventricular activity. 


\subsection{Preprocessing and initial condition}

Since the approach is applied to a single beat at a time, the beats are determined automatically using ECGPUWAVE [7]. The beginning and end of the atrial and ventricular activity are also generated from the ECGPUWAVE method.

In order to have an accurate match between the modeled and original ECG signal, a template matching initial condition is performed. Additionally, a dynamic template is generated for each beat. This choice of the template depends on the sign of the $\mathrm{R}$ peak. This allows greater accuracy during the nonlinear optimization process. The highest cross-correlation point between the initial template and the noisy ECG signal is chosen.

\subsection{Parameter estimation}

The parameter estimation of the proposed model in (4) and (5) is accomplished with the least squares method lsqcurvefit, provided by Matlab [8]. The constraint that the atrial activity occurs prior to that of the ventricular activity is enforced by a partially separable method; since the parameter estimation of the atrial activity is performed separately from the ventricular activity. Additionally, the slopes and magnitudes of difference between two sigmoid functions are bounded greater than zero.

\section{Results}

The proposed approach is applied to the de-noising of ECG signals. The approach is applied to random signals from the LT-ST database, however, the drawback in applying the method to real signals is that there is no quantitave measure of its accuracy; thus, this approach is applied to simulated signals with white, brown and pink noises added to the signal.

\subsection{Application of LT-ST database}

The modelling approach is applied to ischemic beats randomly selected from the LT-ST dataset. Figure 2 show the estimated clean signal for an ischemic beat. Figure 3 shows the error from the original signals versus the clean ones, it can be seen that the error is clinically negligible $(<4 \%)$ [2] compared to the original signal. This error is considered as the noise in the signal. This case study shows the ability of the proposed approach to model ST segments, which are a clinically important feature in ischemia detection. We can see that the modelling approach is able to model the ST change as shown in Figure 2. Further analysis of the error between the original and the clean signal is presented in the simulated case study.

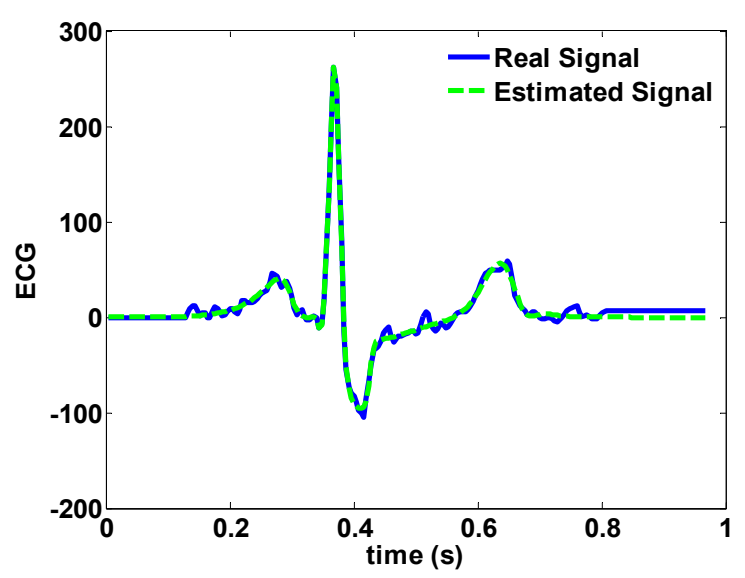

Figure 2: Original healthy and estimated clean signal

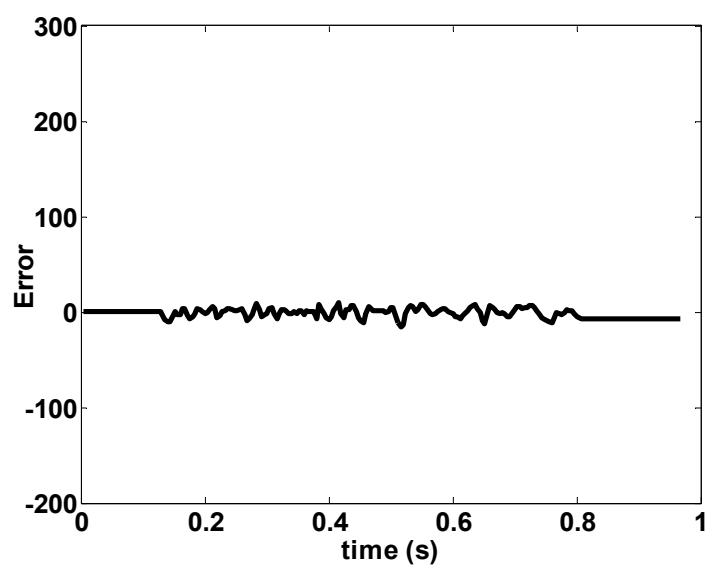

Figure 3: Percentage error between the original and clean signal

\subsection{Simulated signals}

The simulated signals are generated from the model developed in [6]. Additionally, white, brown, and pink noise are added to the signal at an SNR level ranging from -25 to $5 \mathrm{~dB}$ measured at the ST segment [9]. The process is repeated for 40 trials, and the average mean, standard deviation of the error, and $\mathrm{T}$ wave end variation is measured. Figure 4 represents the noisy signal at $-25 \mathrm{db}$ white noise. Additionally, Figure 5 shows the original signal overlapped by the estimated clean signal. It can be see than there is an excellent fit between the two. Table 1 shows the average mean, standard deviation error between the original and estimated signal, and $\mathrm{T}$ wave end variation. It can be seen that the mean error between the estimated clean signal and the original signal is less than $1 \%$, which is clinically negligible. Additionally, the standard deviation of $7 \%$ is less than $10 \%$, which is also negligible in clinical trials. 


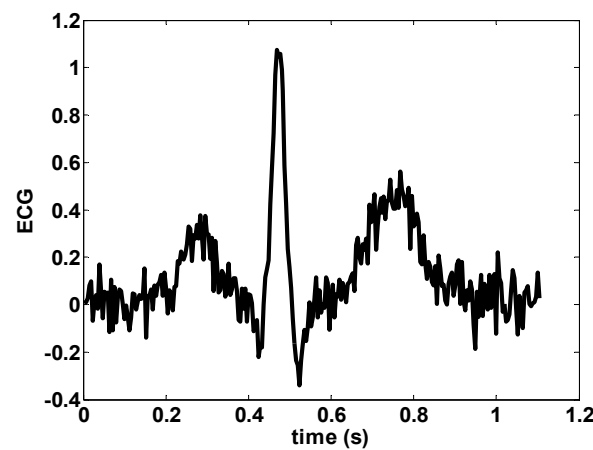

Figure 4 Simulated noisy signal at $-25 \mathrm{~dB}$ at ST segment

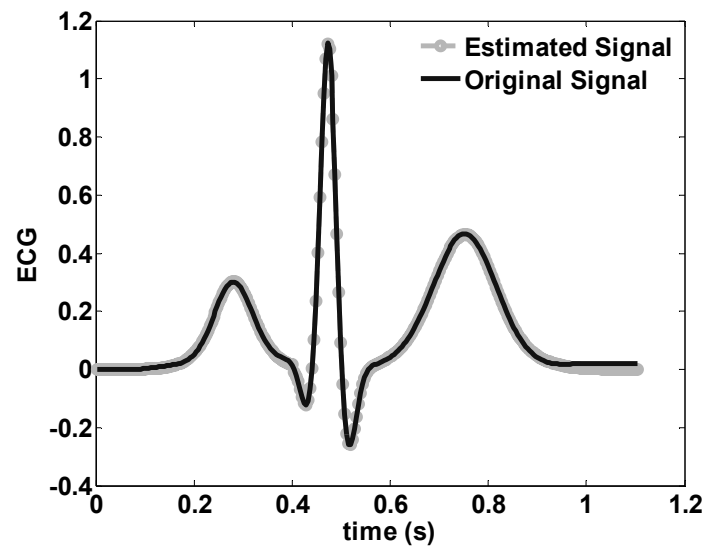

Figure 5 Orignal signal overlapped by clean signal

\begin{tabular}{|c|c|c|}
\hline Noise Type & $\begin{array}{c}\text { Error \% } \\
-25 \mathrm{~dB} \text { to } 5 \mathrm{~dB}\end{array}$ & Twave Var. \% \\
\hline White noise & $\begin{array}{c}0.45 \% \pm 0.48 \% \\
\text { to } 0.11 \% \pm 2.8 \%\end{array}$ & $2.4 \% \pm 1.4 \%$ \\
\hline Pink Noise & $\begin{array}{c}0.1 \% \pm 0.57 \% \text { to } \\
0.54 \% \pm 7.6 \%\end{array}$ & $2.86 \% \pm 1.26 \%$ \\
\hline Brown Noise & $\begin{array}{c}0.11 \% \pm 0.53 \% \\
\text { to } 0.67 \% \pm 4.6 \%\end{array}$ & $1.83 \% \pm 1.03 \%$ \\
\hline
\end{tabular}

Table 1: Error comparison between original and clean signal

\section{Discussion}

This article presented a model based approach for the removal of high frequency noise. The model is based on the cardiac electrophysiological activity. The proposed approach takes into consideration the ECG waves and segment variations with minimal error. Thus, the resulting signal can be used in diagnosis of heart disease without losing clinical information from the original signal.

The drawback behind this approach is that it is applied to a beat by beat process; this can be extended by performing the optimization process on multiple beats attached end-to-end with the real signal.

A cardiac electrophysiological model is presented in this paper for the de-noising of ECG signals. The advantage in addition to the minimal error compared to the clean signal, this approach has the ability to maintain clinical information that helps in heart disease diagnostics such as the ST segment by modeling the late potential that causes the changes in the ST segment.

\section{References}

[1] Daskalov IICaIK. Filtering of electromyogram artifacts from the electrocardiogram. Medical Engineering and Physics 1999, 21:731-736.

[2] GD Clifford AS, PE McSharry, BA Janz. Model-based Filtering, Compression and Classification of the ECG. International Journal of Bioelectromagnetism 2005, Vol. $7: 158-161$

[3] Kestler HAH, M. Kratz, W. Schwenker, F. Palm, G. Hombach, V. Hoher, M. De-noising of high-resolution ECG signals by combining the discrete wavelet transform with the Wiener filter. In Computers in Cardiology; Germany. 1998: 233 - 236.

[4] Zhao LSG. De-Noising of ECG Signal Using TranslationInvariant Wavelet De-Noising Method with Improved Thresholding. In 27th Annual International Conference of the Engineering in Medicine and Biology Society. 2005: 5946 - 5949.

[5] He T. CG, Tarassenko, L.:. Application of Independent Component Analysis in Removing Artefacts from the Electrocardiogram. Neural Comput \& Applic 2006, 15(2):105116.

[6] Patrick E. McSharry GDC, Lionel Tarassenko, and Leonard A. Smith. A Dynamical Model for Generating Synthetic Electrocardiogram Signals. IEEE TRANSACTIONS ON BIOMEDICAL ENGINEERING 2003, 50:289-294.

[7] Goldberger AL AL, Glass L, Hausdorff JM, Ivanov PCh, Mark RG, Mietus JE, Moody GB, Peng CK, Stanley HE. PhysioBank, PhysioToolkit, and PhysioNet: Components of a New Research Resource for Complex Physiologic Signals. AHA Journal Circulation 101 2000, 101:e215e220.

[8] Mathworks. Matlab. 2007.

[9] K. L. Park MJK, B. C. Lee Contact Information, K. S. Jeong, K. J. Lee, and H. R. Yoon. Design of a wavelet interpolation filter for enhancement of the ST-segment. Medical and Biological Engineering and Computing 2001, 39:355-361.

Address for correspondence

Mohamed A. Mneimneh

EECE Department, Marquette University

1515 W. Wisconsin Ave.

Milwaukee, WI 53233

Mohamed.Mneimneh@marquette.edu 